\title{
Performance Improvement of S-shaped Diffuser Using Momentum Imparting Technique
}

\author{
${ }^{1 *}$ Manoj Kumar Gopaliya, ${ }^{2}$ Piyush Jain, ${ }^{3}$ Sumit Kumar, ${ }^{4}$ Vibha Yadav, ${ }^{5}$ Sumit \\ Singh \\ Assistant Professor, Department of Mechanical \& Automobile Engineering, ITM University, Gurgaon (India) \\ Scholars, Department of Mechanical \& Automobile Engineering, ITM University, Gurgaon (India)
}

\begin{abstract}
This paper presents the improvement in performance of $S$-shaped diffuser with rectangular inlet having aspect ratio 2 \& rectangular outlet having area ratio 2 having downstream settling length of $0.5 B$ at Reynolds number $1.37 \times 10^{5}$ with a uniform velocity of $30 \mathrm{~m} / \mathrm{s}$ due to momentum imparting technique. In this technique a cylinder of $0.3 \mathrm{~B}$ diameter is placed at the inflexion plane across the width of the diffuser. Cylinder is rotated at different speed varying from $1500 \mathrm{rad} / \mathrm{s}$ to $4000 \mathrm{rad} / \mathrm{s}$ to impart momentum to the retarded fluid. A computer program based on finite volume technique, using standard $k$-E turbulence model, has been adopted and modified to predict the flow. The results obtained from this study indicate increased pressure recovery accompanied with decrease in non-uniformity in flow at the exit till $3000 \mathrm{rad} / \mathrm{s}$. Beyond this rotational speed, the recovery drops with simultaneous increase in non-uniformity. The pressure recovery of $53.56 \%$ without cylinder improves to $85.53 \%$ with $3000 \mathrm{rad} / \mathrm{s}$ rotational speed of the cylinder. The corresponding decrease in Non-uniformity in flow at the exit is around 2\%. This paper also shows the qualitative representation of secondary flow velocity in the form of contours at the diffuser exit. These variations also support the outcome of this study.
\end{abstract}

Keywords: Effectiveness, Imparting, Momentum, Non-uniformity index, Outlet pressure recovery, S-shaped diffuser.

\begin{tabular}{|llll|}
\multicolumn{2}{c}{ NOMENCLATURE: } \\
\hline$A R$ & Area ratio & $R e$ & $\begin{array}{l}\text { Reynolds number } \\
R e=\rho U_{\text {avgi }} D_{h} / \mu\end{array}$ \\
$A S$ & Aspect ratio & $D_{h}$ & $\begin{array}{l}\text { Hydraulic Diameter at the inlet } \\
\text { where } D_{h}=4 x \text { Area/Perimeter }\end{array}$ \\
$X, Y, Z$ & Cartesian coordinates & & Mass average static pressure at exit \\
$k$ & Turbulent kinetic energy & $P_{s e}$ & Mass average static pressure at inlet \\
& & $P_{s i}$ & \\
$B$ & Width at outlet & $P_{t e}$ & Mass average total pressure at exit \\
$\varepsilon$ & Turbulent kinetic energy dissipation rate & $P_{t i}$ & Mass average total pressure at inlet \\
$\rho$ & Density of working fluid & $S i$ & Non-uniformity index \\
$\mu$ & Viscosity of working fluid & $U_{a v g i}$ & Average velocity at inlet \\
$\xi_{o}$ & Effectiveness of diffuser & $V_{x}$ & Velocity in X-direction \\
$C_{p s}$ & Coefficient of static pressure gain & $V_{y}$ & Velocity in Y-direction \\
$C_{p s e}$ & Coefficient Static pressure gain at exit & $V_{z}$ & Velocity in Z-direction \\
$C_{p s e(i d e a l)}$ & Coefficient of static pressure gain ideally $C_{p s e(i d e a l)}=1-$ & & \\
$C_{p t}$ & $1 / A R 2$ & & \\
$C_{p t e}$ & Coefficient of total pressure loss & & \\
\hline
\end{tabular}

\section{Introduction}

A diffuser is a fluid mechanical device which recovers the pressure energy from the flowing fluid at the expense of its kinetic energy. Diffusers are of many types namely axial, radial and curved depending on the geometry and find very common use in turbo-machinery applications. The wide popularity of curved diffusers in the field of aircraft applications is due to space restrictions and design compatibility. Study of flow parameters within these ducts has been of fundamental interest to researchers in the area of fluid mechanics right from the start of $21^{\text {st }}$ century. Eustine [1] first investigated the nature of flow within the curved ducts and from there on, the continuous, committed and collective efforts of researchers have helped in the understanding of the fluid mechanics within these ducts.

S-shaped diffuser is one of the popular types of curved diffuser. The flow in these diffusers is complicated due to the inflexion in the curvature along the direction of flow, resulting very strong pressure driven stream wise vortices. Many researches have been carried out so far on S-shaped diffuser dealing with issues like effect of different inlet conditions, effect of angle of attack, effect of area ratio, effect of aspect ratio, effect of different inlet and outlet cross-section geometries, effect of angle of turn and many more on pressure 
recovery and non-uniformity of flow through it. In recent research works related to curved diffusers, Majumdar et. al. [2-4] has made detailed measurements on S-shaped rectangular duct having an area ratio 2 with inlet aspect ratio 6 . Anand et al. $[5,6]$ has made experimental investigations on circular $\left(22.5^{\circ} / 22.5^{\circ}\right)$ and rectangular $\left(90^{\circ} / 90^{\circ}\right)$ diffusers having area ratio (AR) 1.9 and 2 respectively. From the start of 21 st century, investigators have also shown keen interest towards automated design and optimization of S-ducts. Zhang et al. [7] has developed an optimization theory for 3-D subsonic S-duct to reduce total pressure distortion and sustain total pressure recovery. Lefantzi and Knight [8] have also developed an automated design optimization process for Sduct taking constraints like airframe weight, space, and line-of-sight blockage of the engine face into account. Apart this, many researchers have significantly contributed in the area of performance-improvement of S-duct by secondary flow control. Reichert and Wendt [9] used fin-type vortex generators for secondary flow control and found considerable improvement in various flow parameters of the duct. Pradeep and Sullerey [10] used vortex generator jets for the above purpose and found $20 \%$ decrease in total pressure loss and flow distortion coefficient in the diffuser. Singh et. al. [11] did some parametric study used for secondary flow control by imparting momentum to the retarding fluid using rotating cylinder at the inflexion plane.

The present research also concentrates towards improving the performance of the diffuser using momentum imparting technique adopted by Singh et. al. [11]. But the inlet flow velocity and range of rotational speed of cylinder are different. Apart this, the present paper also shows the effect of rotating cylinder on the uniformity of exit flow through diffuser which indeed is one of the basic flow requirement to the compressor of the fighter aircraft along with pressure rise. Hence the present paper gives a much better ground to judge the merit of momentum imparting technique in context of secondary flow control in curved diffusers.

\section{Geometrical Description And Computational Fluid Dynamics Analysis Of S-Shaped} Diffuser

\subsection{Geometrical parameters}

In present research work, the S-shaped diffuser examined is having rectangular inlet with aspect ratio 2 and rectangular outlet cross-section with area ratio 2 having downstream settling length of $0.5 \mathrm{~B}$ which is provided to allow the flow to get settle down after second curvature or bend. The schematic layout S-shaped diffuser is shown in Figure (1).

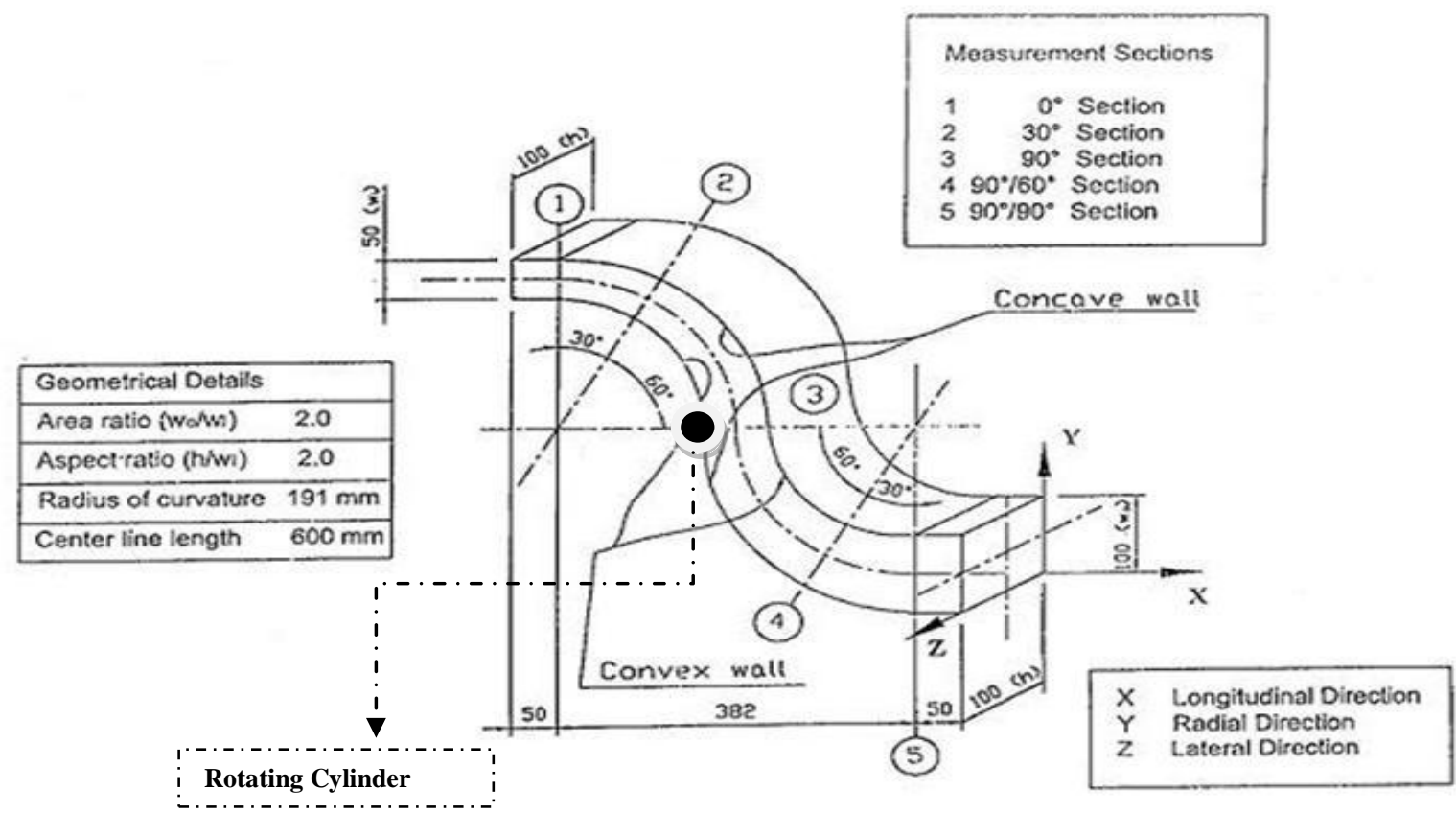

Figure (1): Schematic layouts of S-shaped diffusers under study (Ref. 5)

It is having $90^{\circ} / 90^{\circ}$ of turning in both upper and lower limb. Its radius of curvature is $1.91 \mathrm{~B}$ and centerline length is $6.0 \mathrm{~B}$. Its inlet cross-section is rectangular with $0.5 \mathrm{~B}$ depth and $1.0 \mathrm{~B}$ width. Here $\mathrm{B}$ is the outlet width of rectangular cross-section whose value is $100 \mathrm{~mm}$, obtained taking area ratio 2 . Firstly, offset is given to the duct in the horizontal plane in the form of $90^{\circ}$ turn in both upper and lower limbs. After that, a single cylinder having $0.3 \mathrm{~B}$ diameter is placed across the width of the diffuser at inflexion plane. Approximately, $115^{\circ}$ sector of cylinder is in touch with the flow inside the duct [11]. 


\subsection{Description of geometric model generation and computational tools used}

The geometric model has been created in CATIA V5R17 [12] modeling software with dimensions described in para. 2.1. S-duct has been made by using surfacing technique with the help of multi sections solid command. This shape verifies the continuity well and best fitted the curved geometries. Special care has been taken for the regions where higher gradients in field variable had been observed. The software HYPERMESH V8 [13] has been used for the meshing of the duct. Five boundary layers with the thickness of first layer as $0.5 \mathrm{~mm}$ and a growth rate of 1.2 afterwards have been adopted.

A commercially available CFD code "FLUENT 6.3" [14] has been used for the analysis of flow through this diffuser. The governing equations [15] for mean flow in reduced form for steady incompressible flows are:

$$
\begin{gathered}
\frac{\partial}{\partial x_{i}}\left(\rho u_{i}\right)=S_{m} \\
\rho u_{j} \frac{\partial u_{i}}{\partial x_{j}}=-\frac{\partial p}{\partial x_{i}}+\frac{\partial}{\partial x_{j}}\left[\mu\left(\frac{\partial u_{i}}{\partial x_{j}}+\frac{\partial u_{j}}{\partial x_{i}}\right)-\frac{2}{3} \mu \delta_{i j} \frac{\partial u_{i}}{\partial x_{i}}\right]+\frac{\partial}{\partial x_{j}}\left(-\overline{\rho u_{i}^{\prime} u_{j}^{\prime}}\right)
\end{gathered}
$$

These equations are of the same general form as the original equations except for some additional terms which are the Reynolds stresses. These need to be modelled for closure solution. In this research, standard k- $\varepsilon$ model with boundary conditions prescribed in table (1) has been adopted for this purpose. To calculate the Reynolds stresses with the standard k- $\varepsilon$ model, an extended Boussinesq relationship is used as follows:

$$
\overline{-\rho u_{i}^{\prime} u_{j}^{\prime}}=\mu_{t}\left(\frac{\partial u_{i}}{\partial x_{j}}+\frac{\partial u_{j}}{\partial x_{i}}\right)-\frac{2}{3}\left(\rho k+\mu_{t} \frac{\partial u_{i}}{\partial x_{i}}\right) \delta_{i j}
$$

where $\mathrm{k}$ is the turbulent kinetic energy, $\delta_{\mathrm{ij}}$ is the Kronecker delta and $\mu_{\mathrm{t}}$ is the eddy viscosity.

$\mu_{\mathrm{t}}$ is calculated as follows:

$$
\mu_{t}=\rho C_{\mu} k^{2} / \varepsilon
$$

The additional equations for $\mathrm{k}$ and $\varepsilon$ for steady incompressible flow in simplified form are:

$$
\begin{array}{r}
\rho u_{i} \frac{\partial k}{\partial x_{i}}=\frac{\partial}{\partial x_{i}}\left[\left(\mu+\frac{\mu_{t}}{\sigma_{k}}\right) \frac{\partial k}{\partial x_{i}}\right]+G_{k}-\rho \varepsilon+Y_{M} \\
\rho u_{i} \frac{\partial \varepsilon}{\partial x_{i}}=\frac{\partial}{\partial x_{i}}\left[\left(\mu+\frac{\mu_{t}}{\sigma_{\varepsilon}}\right) \frac{\partial \varepsilon}{\partial x_{i}}\right]+C_{1 \varepsilon} \frac{\varepsilon}{k} G_{k}-C_{2 \varepsilon} \rho \frac{\varepsilon^{2}}{K}
\end{array}
$$

$\mathrm{G}_{\mathrm{k}}$ represents the production of turbulent kinetic energy and is evaluated as:

$$
G_{k}=\mu_{t} S^{2}
$$

where $\mathrm{S}$ is the modules of mean rate of strain tensor defined as:

Here mean strain rate $S_{i j}$ given by:

$$
S=\sqrt{2 S_{i j} S_{i j}}
$$

$$
S_{i j}=\frac{1}{2}\left[\frac{\partial u_{i}}{\partial x_{j}}+\frac{\partial u_{j}}{\partial x i}\right]
$$

The dilatation dissipation term $\left(\mathrm{Y}_{\mathrm{M}}\right)$ has been neglected for incompressible flow. The value of the empirical constants used are $\mathrm{C}_{1 \varepsilon}=1.44, \mathrm{C}_{2 \varepsilon}=1.92, \mathrm{C}_{\mu}=0.09, \sigma_{\mathrm{k}}=1.0$ and $\sigma_{\varepsilon}=1.33$. These values have been found to work reasonably well for a wide range of wall bounded and free shear flows. 
Flow simulations in FLUENT were carried out adopting a cell centred finite volume approach to solve the Partial Differential Equation using segregated solver. An implicit solution scheme in conjunction with an algebraic multi-grid (AMG) method was used for faster convergence. The second-order upwind scheme was used in the discretization scheme for all equations to achieve higher accuracy. Coupling between velocity and pressure was established by pressure velocity correlation using SIMPLE algorithm. Under relaxation factors were used for all equations to satisfy the Scarborough condition for convergence of the solutions and also avoiding the divergence in the solutions. Convergence of the solution was assumed when sum of normalized residual for each conservation equation was less than or equal to $10^{-6}$. The residuals for different equations are given as:

$$
\frac{\sum_{i=1}^{M}\left|R_{i}\right|^{n}}{S_{N \phi}} \leq 10^{-6}
$$

where, $\llbracket \boldsymbol{R}_{\boldsymbol{i}} \|^{\boldsymbol{n}}$ is the sum of the absolute residuals for a dependent variable $\phi$ for the $\mathrm{n}^{\text {th }}$ iteration and $\mathrm{S}_{\mathrm{N} \phi}$ the corresponding normalizing factor.

\subsection{Performance parameters}

The important performance evaluation parameters are:

a.) Coefficient of static pressure gain: It represents the extent by which kinetic energy has been converted into pressure energy due to diffusing action at any location (as $\mathrm{x})$.

$$
C_{p s}=2\left(P_{s x}-P_{s i}\right) / \rho U^{2} \text { avg }
$$

b.) Effectiveness of diffuser: It represents the pressure recovery capacity of diffuser in comparison with ideal diffuser.

c.) Coefficient of total pressure loss:

$$
\xi_{o}=C_{p s e} / C_{p s e(i d e a l)}
$$

$$
C_{p t}=2\left(P_{t i}-P_{t x}\right) / \rho U_{a v g}^{2}
$$

d.) Non-uniformity index: It represents the percentage amount of secondary flow at any location.

$$
S i=\left(\sum \operatorname{sqrt}\left(V_{y}^{2}+V_{z}^{2}\right)\right) /\left(n * U_{\text {avg }}\right)
$$

Here "summation sign" includes all the grid point in the outlet plane and " $n$ " is its numerical value.

\subsection{Convergence Criteria and Validation of CFD Code}

The model and boundary conditions shown in Table 1 have been validated with the experimental values obtained by Anand et al. [5] which are in good agreement.

Table 1: Description of boundary conditions

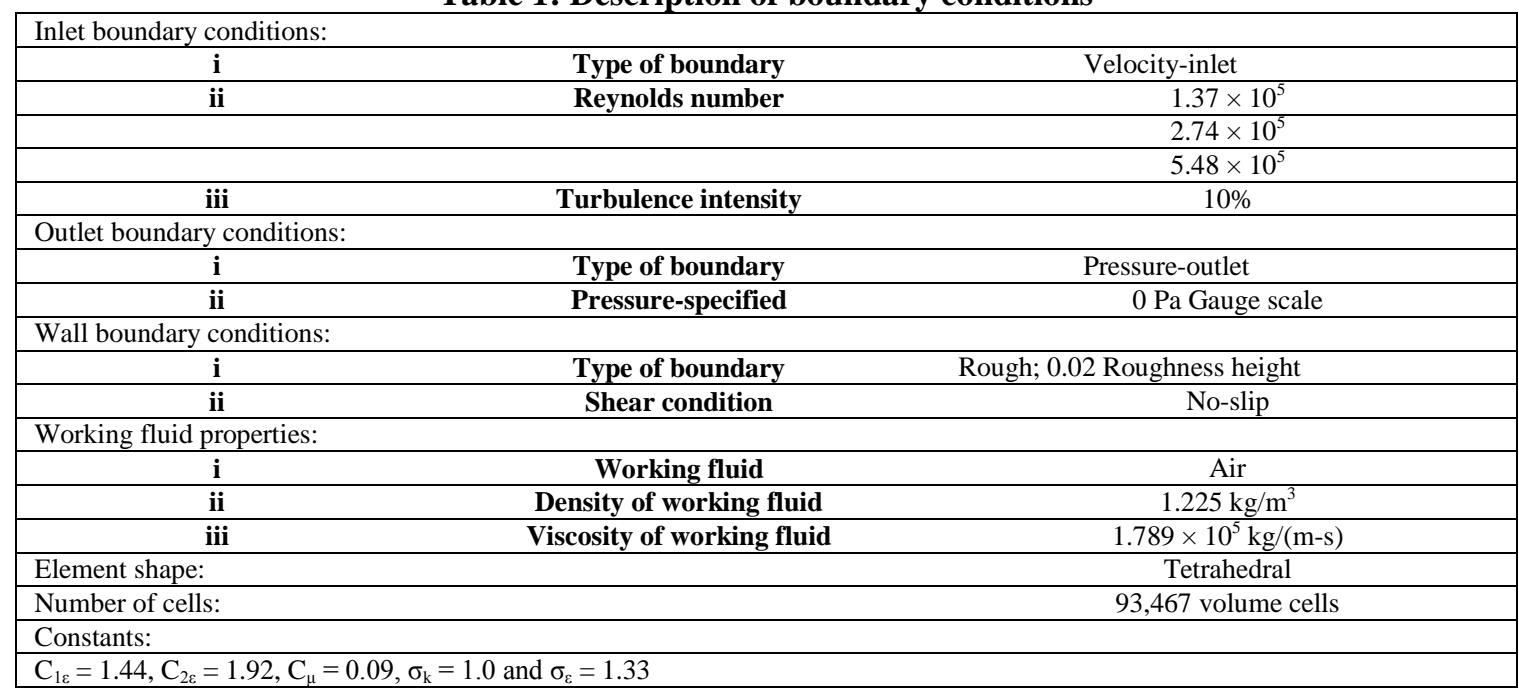

Table 2 shows comparison of experimental and computational values of $\mathrm{C}_{\mathrm{pse}} \& \mathrm{C}_{\mathrm{pte}}$ for three Reynolds numbers. 
Table 2 (a): Experimental \& Computational Values of $\mathbf{C}_{\mathrm{ps}}$ for different Reynolds Numbers

\begin{tabular}{|llll|}
\hline Reynolds No. & $\begin{array}{l}\mathrm{C}_{\mathrm{pse}}(\text { Experimental) } \\
\text { in } \%\end{array}$ & $\begin{array}{l}\mathrm{C}_{\mathrm{pse}} \text { (Computational) } \\
\text { in \% }\end{array}$ & \%age error \\
\hline $\mathbf{1 . 3 7 e + 0 5}$ & $\mathbf{5 5 . 3 3}$ & $\mathbf{5 3 . 5 6}$ & $3.20 \%$ \\
\hline $\mathbf{2 . 7 4 e + 0 5}$ & $\mathbf{5 8 . 6 7}$ & $\mathbf{5 5 . 5 3}$ & $5.35 \%$ \\
\hline $\mathbf{5 . 4 8 e + 0 5}$ & $\mathbf{6 0 . 0 0}$ & $\mathbf{5 7 . 5 3}$ & $4.12 \%$ \\
\hline
\end{tabular}

Table 2(b): Experimental \& Computational Values of $\mathbf{C}_{\mathrm{pt}}$ for different Reynolds Numbers

\begin{tabular}{|llll|}
\hline Reynolds No. & $\begin{array}{l}\mathrm{C}_{\text {pte }}(\text { Experimental }) \\
\text { in } \%\end{array}$ & $\begin{array}{l}\mathrm{C}_{\text {pte }}(\text { Computational }) \\
\text { in \% }\end{array}$ & \%age error \\
\hline $\mathbf{1 . 3 7 e + 0 5}$ & $\mathbf{2 0 . 1 0}$ & $\mathbf{2 0 . 6 0}$ & $2.49 \%$ \\
\hline $\mathbf{2 . 7 4 e + 0 5}$ & $\mathbf{1 8 . 8 9}$ & $\mathbf{1 9 . 5 8}$ & $3.52 \%$ \\
\hline $\mathbf{5 . 4 8 e + 0 5}$ & $\mathbf{1 7 . 9 1}$ & $\mathbf{1 8 . 6 1}$ & $3.76 \%$ \\
\hline
\end{tabular}

It was shown by Gopaliya et al. [16] in the study of combined offset effect using the same diffuser that among two very popular turbulent models i. e. Standard $\mathrm{k}-\varepsilon$ model and Standard $\mathrm{k}-\omega$ model, later gives higher deviations from experimental data during flow analysis through the duct. This is due to the fact that the $\mathrm{k}-\omega$ turbulent model produces best results for low Reynolds number flows; however the Reynolds numbers involve in present study are very high. Thus standard $\mathrm{k}-\varepsilon$ turbulence model has been chosen in the present.

The details regarding wall pressure distribution, location of separation \& concentration of secondary flow throughout the diffuser have not been presented in here. However, during validation process these aspects were kept in mind and therefore the meshing intensity close to the walls were increased to resolve the lower sub-layer $(\mathrm{y}+)$ values and also by doing additional grid independency tests in the core regions throughout second limb where velocity gradients were steeper.

The deviations have not been resolved completely, which may be attributed to the fact that the flow is highly 3D and the uncertainty in the experimental values which may largely be due to the use of 3 hole probe which can only measure 2-dimensional plane velocity. The second reason could be that the $k$ - $\varepsilon$ turbulence model has its limitation in resolving the flow in the region having sharp variations in velocity gradient.

$\mathrm{S}$-shaped diffuser with rectangular outlet used in present research work is same as Anand et al. [5]. Hence, the above discussed validation work is sufficient for present research.

Figure (2) shows the comparison of experimental and computational values of $\mathrm{C}_{\mathrm{ps}}$ and $\mathrm{C}_{\mathrm{pt}}$ along the centreline of the S-shaped diffuser for three different Reynolds number.

$\operatorname{For} \operatorname{Re}=1.37 \mathrm{e}+05$

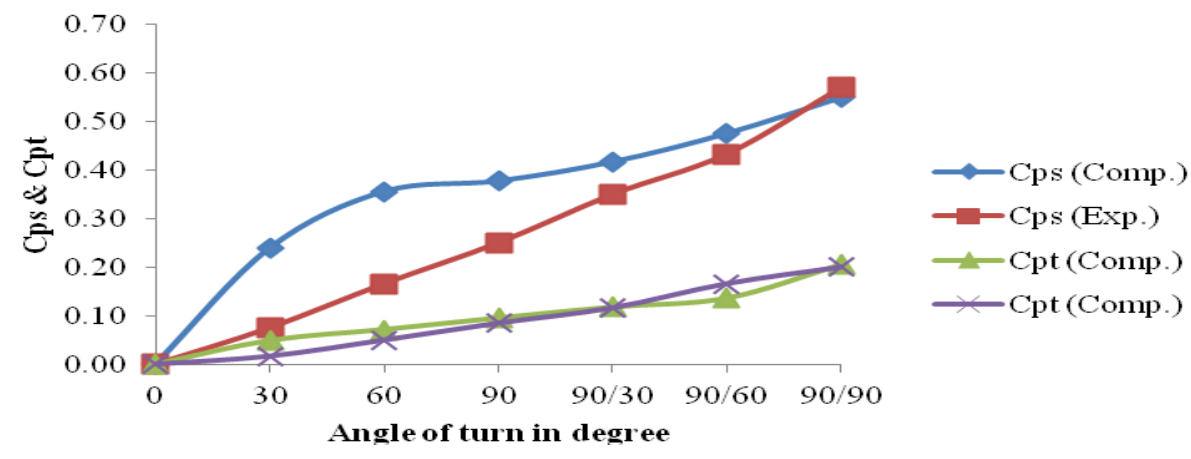

For $\operatorname{Re}=2.74 \mathrm{e}+05$

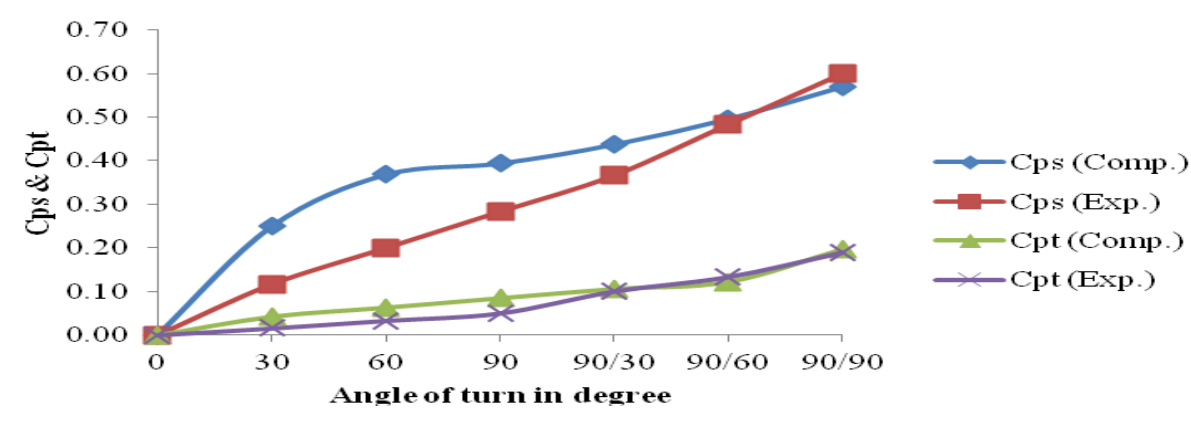




\section{For $\operatorname{Re}=5.48 \mathrm{e}+05$}

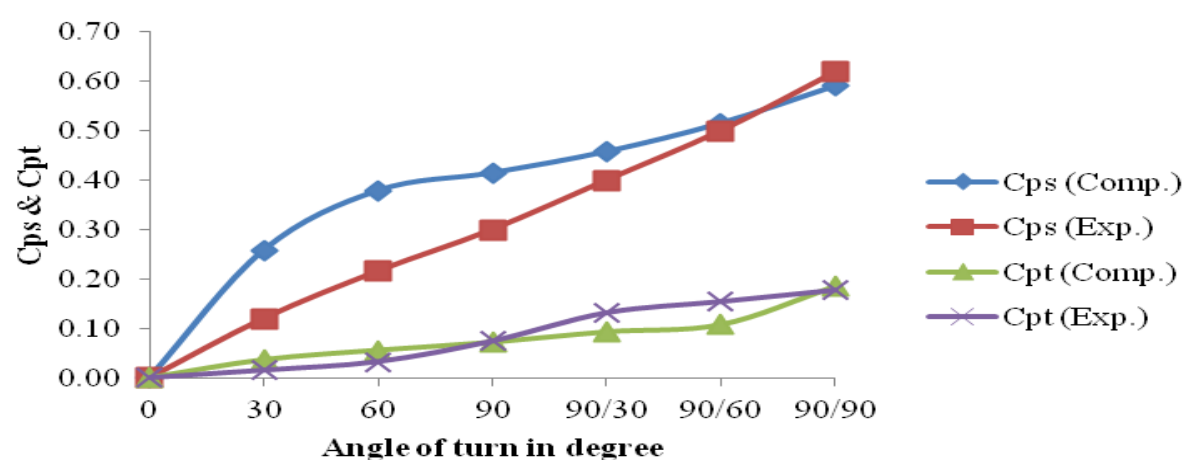

Figure (2): Comparison of experimental and computational values of $C_{p s}$ and $C_{p t}$ along the centre line of the $\mathrm{S}$-shaped diffuser for three different Reynolds numbers.

\section{Results and discussions}

In the present research, effect of momentum imparting on different performance characteristics of Sshaped diffuser has been studied. For this, the cylinder placed across the width of the S-shaped diffuser at inflexion plane is rotated at different speed ranges from $1500 \mathrm{rad} / \mathrm{s}$ to $4000 \mathrm{rad} / \mathrm{s}$. This rotation imparts momentum to the retarding flow and helps in controlling secondary flow in the flow at inflexion plane and further.

In the present research work, analysis has been performed for three Reynolds numbers viz. $1.37 \times 10^{5}$, $2.74 \times 10^{5}$ and $5.48 \times 10^{5}$ corresponding to uniform inlet velocity of $30 \mathrm{~m} / \mathrm{s}, 60 \mathrm{~m} / \mathrm{s}$ and $120 \mathrm{~m} / \mathrm{s}$. Since the outcomes are of similar trends for all three Reynolds numbers and to avoid duplication of data, the data for Reynolds number $1.37 \times 10^{5}$ only has been presented.

\subsection{Grid independency check}

Grid (Mesh) independency check is the foremost important and necessary step while doing analysis of any CFD problem using software or code because of the great dependency of solution on the fineness of meshing. Mesh (3) as in Table (3) has been chosen as final meshing, since CFD outcome has become fairly constant beyond this grid density.

Table 3: Details of Grid Independency Check

\begin{tabular}{|lll|}
\hline S. No. & No. of Volume Cells & $\mathrm{C}_{\mathrm{pse}}(\%)$ \\
\hline 1. & $\mathbf{7 4 , 5 6 7}$ & $\mathbf{5 4 . 3 4}$ \\
\hline 2. & $\mathbf{7 9 , 1 9 0}$ & $\mathbf{5 4 . 0 9}$ \\
\hline 3. & $\mathbf{9 3 , 4 6 7}$ & $\mathbf{5 3 . 5 6}$ \\
\hline 4. & $\mathbf{1 , 0 7 , 2 5 3}$ & $\mathbf{5 3 . 6 4}$ \\
\hline
\end{tabular}

In present research, efforts have been made to create geometric model using CATIA V5R17[12] modeling software which is much advanced modeling tool as compared to commonly used modeling tools in CFD such as GAMBIT. Apart this, HYPERMESH V8 [13] has been used for the meshing of the duct with tetrahedral shaped elements. This has helped in reducing the total number of elements required to get optimum accuracy during validation.

\subsection{Effect of momentum imparting on coefficient of static pressure gain $\left(C_{p s}\right)$ along centre line}

Momentum imparting at inflexion plane due to rotation of cylinder results in improvement in pressure recovery beyond inflexion plane. Increase in rotational speed till $3000 \mathrm{rad} / \mathrm{s}$ enhances the pressure recovery at all locations beyond inflexion point by providing much needed energy support to the retarding fluid layers.

Further increase in rotational speed results in decrease in pressure recovery beyond $90^{\circ} / 60^{\circ}$ location. This is due to excessive intermixing of fluid layers at inflexion plane beyond $3000 \mathrm{rad} / \mathrm{s}$ which reaches down the flow line near outlet. This results in local increase in secondary flow at all locations beyond inflexion plane. Figure (3) shows these trends for $\operatorname{Re}=1.37 \times 10^{5}$. 


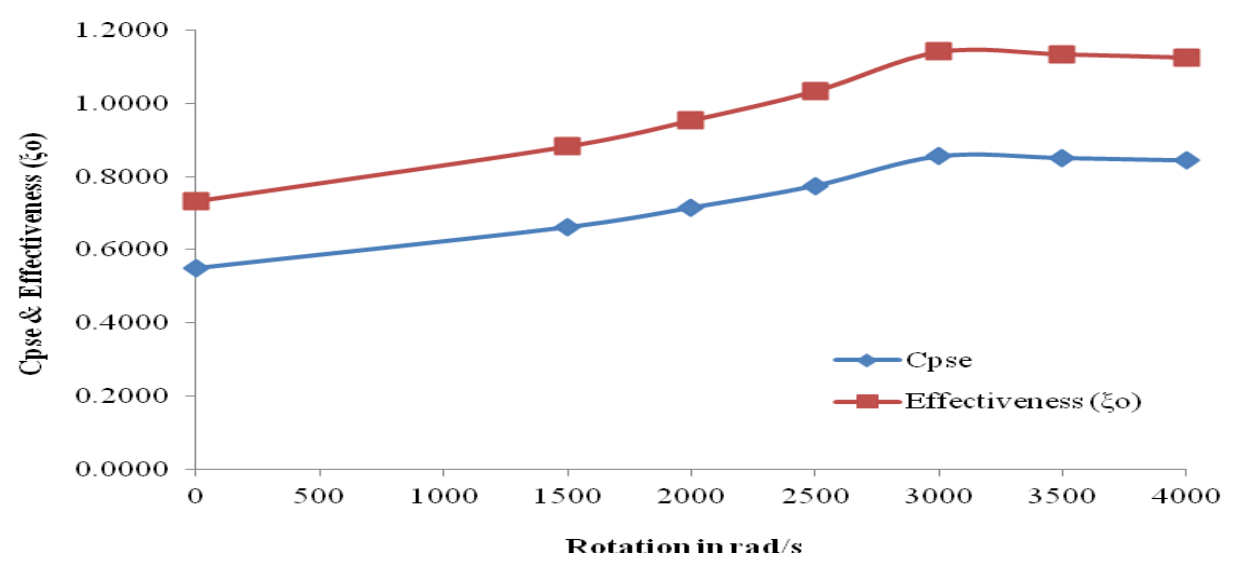

Figure (3): Variation in $\mathrm{C}_{\mathrm{ps}}$ along the centre line of S-Shaped Diffuser with rotational speed of cylinder

\subsection{Effect of momentum imparting on coefficient of static pressure gain at exit ( $\left.C_{p s e}\right)$}

Momentum imparting at inflexion plane results in increase in coefficient of static pressure gain at exit and its value goes on increasing with rotational speed till $3000 \mathrm{rad} / \mathrm{s}$, beyond that it drops. Reasoning for these trends is already being explained in para. 3.2.

This effect can also be represented in terms of effectiveness $\left(\xi_{0}\right)$ of diffuser, which also shows the similar trends as $\mathrm{C}_{\mathrm{pse}}$. In present case, $\mathrm{AR}=2$, therefore $\mathrm{C}_{\mathrm{pse}(\mathrm{ideal})}=75 \%$. Figure (4) shows these variations in graphical form at $\operatorname{Re}=1.37 \times 10^{5}$.

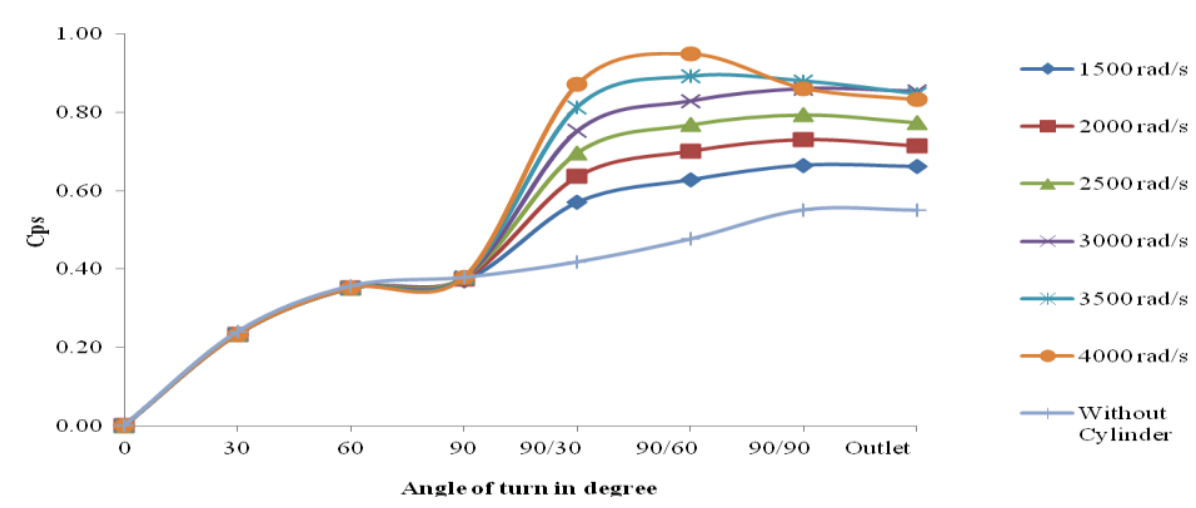

Figure (4): Variation of $\mathrm{C}_{\mathrm{pse}}$ and Effectiveness $\left(\xi_{\mathrm{o}}\right)$ of S-Shaped diffuser with speed of rotation of cylinder at Reynolds number $1.37 \times 10^{5}$

\subsection{Effect of momentum imparting on uniformity of flow at outlet}

S-shaped diffusers are the member of curved diffusers family which find wide utility in fighter aeroplanes, aircraft etc., where flow non-uniformity needs greater concern and to be kept within limit for satisfactory performance. Effect of non-uniformity is represented by percentage secondary flow at outlet of the diffuser. Imparting of momentum with the help of rotating cylinder at inflexion plane results in $1.5 \%$ to $2 \%$ improvement in uniformity of flow till 3000rad/s. Beyond this speed, imparted momentum deteriorate the uniformity of flow which is due the increased localized intermixing after inflexion plane due to higher rotation speed. This argument is well supported by the fact that pressure recovery also drops beyond this point. Figure (5) shows these variations in graphical form at $\mathrm{Re}=1.37 \times 10^{5}$. 


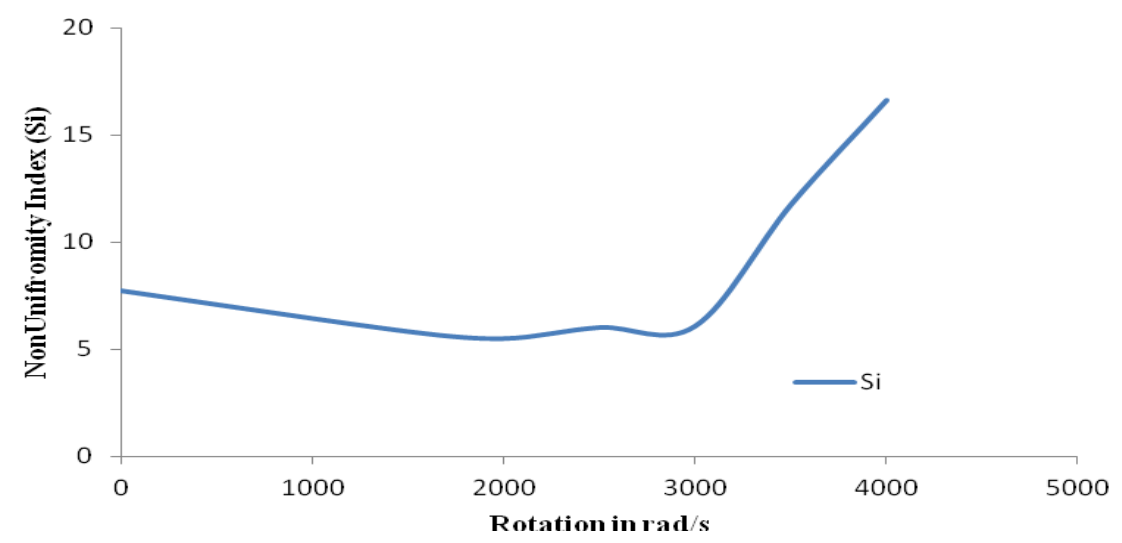

Figure (5): Variation of Non-Uniformity Index (Si) of S-Shaped diffuser with speed of rotation of cylinder

\subsection{Qualitative analysis}

at Reynolds number $1.37 \times 10^{5}$

Velocity contours are much better way of representing variations in different flow parameters in any parametric study. Figures (6) shows non-dimensionalized secondary flow contours at the exit of the diffuser with different rotational speed. With the analysis of these contours, it can be concluded that secondary flow contours at diffuser exit progressively become more uniform with induction of momentum due to cylinder rotation. This continues quite fairly till $3000 \mathrm{rad} / \mathrm{s}$ beyond which contours start distorting. This is in accordance with the variation in the value of non-uniformity index mentioned above and hence supports the argument.

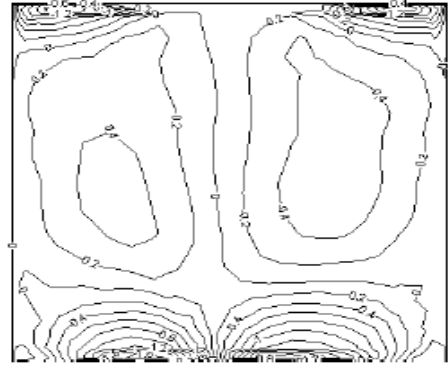

Without rotation

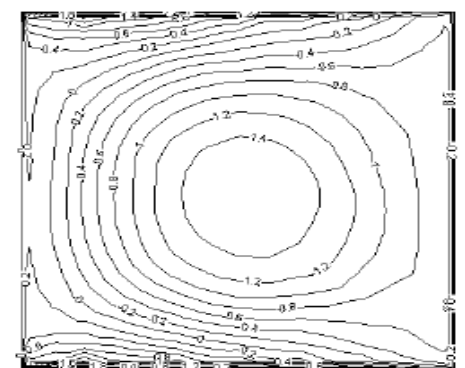

$1500 \mathrm{rad} / \mathrm{s}$
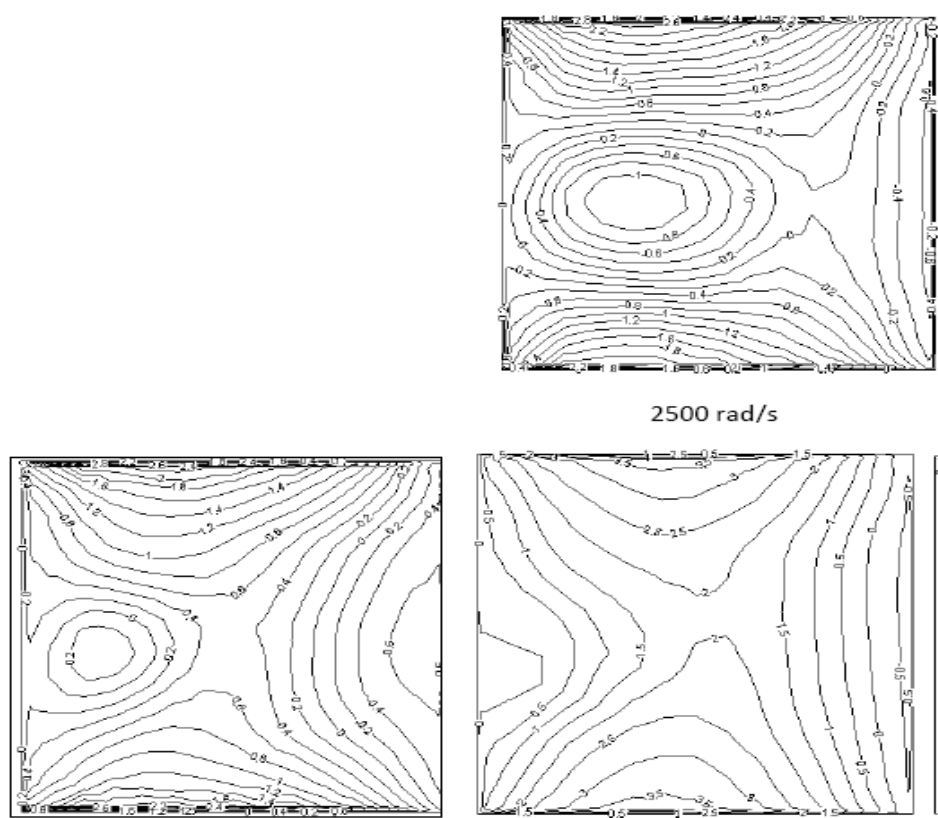

$3000 \mathrm{rad} / \mathrm{s}$
$2500 \mathrm{rad} / \mathrm{s}$

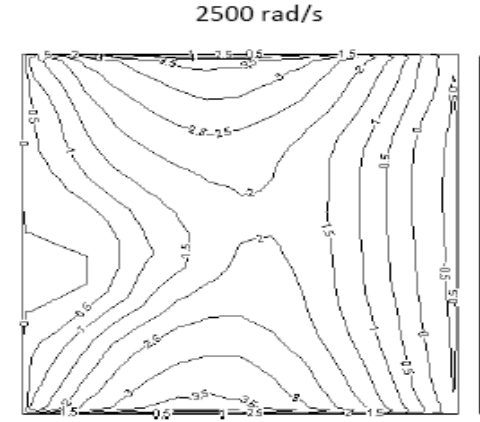

$3500 \mathrm{rad} / \mathrm{s}$

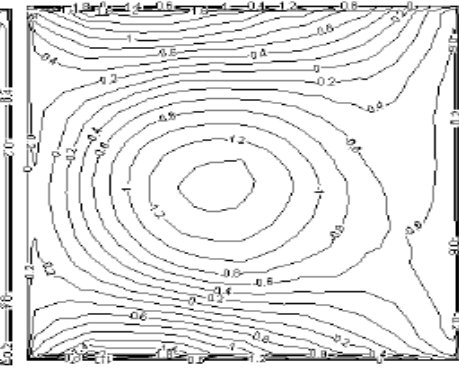

$2000 \mathrm{rad} / \mathrm{s}$

Figure (6): Secondary Flow Contours at diffuser exit for different rotational speed at Reynolds number $1.37 \times 10^{5}$ 


\section{Conclusions}

In the present research, effect of momentum imparting on different performance characteristics of Sshaped diffuser has been studied. The momentum is imparted by placing a rotating cylinder across the width of the S-shaped diffuser at inflexion plane. The rotational speed of the cylinder is varied from $1500 \mathrm{rad} / \mathrm{s}$ to 4000 $\mathrm{rad} / \mathrm{s}$. Imparted momentum energises the retarding flow and helps in controlling secondary flow beyond inflexion plane.

The following conclusions have been drawn from the present study:

i. Momentum imparting results in increase in coefficient of static pressure gain at exit $\left(\mathrm{C}_{\mathrm{pse}}\right)$ through the S-shaped diffusers. Its value increases from $54.97 \%$ for diffuser without cylinder to $85.54 \%$ for diffuser with cylinder rotating at 3000 rad/s. Beyond this speed, its value starts decreasing.

ii. Non-uniformity decreases from $7.72 \%$ for diffuser without cylinder by 1.5 to $2 \%$ till $3000 \mathrm{rad} / \mathrm{s}$. Any further increase in rotation speed results in steep rise in non-uniformity in flow at diffuser exit.

iii. Non-dimensional secondary flow velocity contours progressively become more uniform with increase rotational speed of cylinder till 3000rad/s. Distortion of contours starts with any further increase in rotational speed beyond 3000rad/s.

iv. The optimum speed of cylinder rotation in present case is come out to be 3000rad/s. At this speed, coefficient of static pressure gain at exit $\left(\mathrm{C}_{\mathrm{pse}}\right)$ is $85.54 \%$ with $6.06 \%$ of flow non-uniformity at diffuser exit.

The improved pressure recovery of diffuser due to momentum imparting technique is also accompanied with increased power requirement of the fighter aircraft on account of rotating cylinder. Hence the overall improvement in the functioning of fighter aircraft due to momentum imparting technique at diffuser can only be judged by assessing the net gain in the power consumption of the aircraft due to these two opposite aspects.

\section{References}

[1] Eustine, J., Flow of Water in Curved Pipes, Proc. Royal Society, London, Series A - 84, 1910, pp.107-118.

[2] Majumdar, B., Flow Investigation in Curved Diffuser, Ph.D. Thesis, Department of Applied Mechanics, Indian Institute of Technology Delhi, New Delhi, India, 1994.

[3] Majumdar, B.; Singh, S. N; and Agrawal, D. P., Flow Characteristics in S-Shaped Diffusing Duct, Int. Journal of Journal Turbo and Heat Engines, vol. (14), 1997, pp. 45-47.

[4] Majumdar, B.; Ratan Mohan; Singh, S. N; and Agrawal, D. P., Experimental Study of Flow in High Aspect Ratio $90^{\circ}$ Curved Diffuser, Trans. ASME., Journal of Fluid Engineering, vol. (120), 1998, pp. 83-89.

[5] Anand, R. B.; Lajpat Rai; Singh, S. N.; and Sharma O. P., Flow Characteristics of A Low Aspect Ratio $90^{\circ} / 90^{\circ}$ S-Shaped Diffuser, Journal of Aeronautical Society of India vol. (53), 2001, pp. 239-252.

[6] Anand, R. B.; Lajpat Rai; and Singh, S. N., Flow and Performance Characteristics of $22.5^{\circ} / 22.5^{\circ}$ S-Shaped Circular Diffuser, Proc. of $28^{\text {th }}$ national conference on FMEP, Chandigarh, India, 2001, pp. 364-371.

[7] Zhang, W.; Knight, D.; and Smith, D., Automated Design of A Three Dimensional Subsonic Diffuser, Journal of Propulsion and Power vol. (16), No. 6, 2000, pp. 1132-1140.

[8] Lefantzi, S.; and Knight, D., Automated Design Optimization of A Three Dimensional S-Shaped Subsonic Diffuser, Journal of Propulsion and Power vol. (18), No. 4, 2002, pp. 913-921.

[9] Reichert, B. A.; and Wendt, B. J., Improving Diffusing S-Duct Performance by Secondary Flow Control, NASA Lewis Research Center, Cleveland, Ohio, AIAA Journal, 1994.

[10] Pradeep, A. M.; and Sullerey, R. K., Secondary Flow Control in A Circular S-Duct Diffuser Using Vortex Generator Jets, 2nd AIAA Flow Control Conference, 28 June-1 July, Portland, Oregon, 2004.

[11] Singh, S. N.; Seshadri, V.; Chandel Sunil; and Gaikwad Mahendra, Analysis of the Improvement in Performance Characteristics of S-Shaped Rectangular Diffuser by Momentum Imparting Using Computational Fluid Dynamics, Engineering Applications of Computational Fluid Mechanics Vol. 3, No. 1, 2009, pp. 122-135.

[12] Dassault Systèmes India Pvt. Ltd. CATIA V5R17.

[13] Altair Engineering India Pvt. Ltd. HYPERMESH V8.

[14] Active Bangalore (2009). FLUENT 6.3 User Guide, Vol. 1-4.

[15] Launder BE; and Spalding DB, The numerical computation of turbulent flow, Comp. Methods App. Mech. Engineering vol. (3), 1974, pp. 269-289.

[16] Gopaliya, M. K.; Goel Piyush; Prashar Sunil; Dutt Anil; CFD analysis of performance characteristics of S-shaped diffusers with combined horizontal and vertical offsets, Computers \& Fluids, Elsevier Publications vol. (40), 2011, pp. 280-290. 